\title{
Implementing Best Practices at the National Center for Cryo-EM Access and Training
}

Edward Eng, Anchi Cheng, Hui Wei, Mahira Aragon, Cathleen Castello, Elina Kopylov, Clint Potter and Bridget Carragher

\section{New York Structural Biology Center, New York, New York, United States}

To broaden biomedical scientists' access to cryogenic electron microscopy (cryoEM), the NIH Common Fund's Transformative High-Resolution Cryo-Electron Microscopy program has created three national service centers to provide access to cryoEM technology and the development of training curricula to build a skilled workforce. The mission of the National Center for CryoEM Access and Training (NCCAT) is twofold: to provide nationwide access to advanced cryoEM technical capabilities, and to assist users in the development of cryoEM skills needed for independent research. NCCAT provides access to state-ofthe-art equipment required to solve structures to the highest possible resolution using cryoEM methods.

Advances in imaging technology and data processing have resulted in the recent surge in interest in solving structures using cryoEM, which can now extend to near-atomic resolution. In particular, detector improvements and related software, now give us the ability to capture information from vitrified biological macromolecules nearly to the Nyquist limit, and improved lens stability allows maintenance of good alignments over the entire data collection period, which can extend for several days. While the instrumentation is outstanding, sample preparation remains a significant bottleneck to the generation of high-resolution structures. Standard blot-vitrification techniques require expertise to master and even so can result in inconsistent sample quality. To address the reproducibility of sample preparation, NCCAT provides access to a novel method for vitreous ice grid preparation robot $[1,2]$ that dispenses nL volumes of sample onto a "self-blotting" grid [3]. A prototype of a commercial version of this device (Chameleon from TTP Labtech, Inc.) is available for access. The system also provides some advantages in ameliorating the deleterious effects of particles [4] coming into contact with the air-water interface during the process of forming a thin layer suitable to vitrification and EM imaging.

Increasing data throughput is an ongoing goal, both in the general case to improve access to scarce instrumentation, as well as for specific samples where sub-optimal sample distribution, or stability issues, may mean that very large numbers of images need to be collected in order to acquire enough intact particle views to contribute to a high-resolution $3 \mathrm{D}$ reconstruction. To increase the throughput of data collection, NCCAT has implemented large image shift data collection through the Leginon data acquisition platform $[5,6]$; and induced coma and astigmatism can be reduced to allow for image shifts $>15 \mu \mathrm{m}$ from the optical axis. Under these conditions, images can be acquired with a turnaround of $~ 20$ s (on a K2 direct detector)

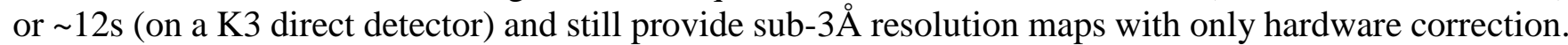
For more conservative data collection strategies, moderate image shift configurations may be chosen to routinely achieve $2 \AA$ reconstructions without the need for additional software image correction. Although utilizing software correction ameliorates many of these aberrations the goal of the center is to provide as high-quality data as efficiently as possible. By implementing the most current best practices users are able to optimize different experimental parameters on-the-fly, thereby allowing reconstructions to be determined from images collected in a single day.

Our objective is to enable biomedical researchers from all fields to make use of cryoEM techniques and methodologies in their research programs [7].

\section{References}


[1] Razinkov et al. A new method for vitrifying samples for cryoEM. J Struct Biol. 2016;195(2):190-8.

[2] Dandey et al. Spotiton: New features and applications. J Struct Biol. 2018.

[3] Wei et al. Optimizing "self-wicking" nanowire grids. J Struct Biol. 2018.

[4] Noble AJ et al. Reducing effects of particle adsorption to the air-water interface in cryoEM. Nature Methods 10 (2018), p 793.

[5] C. Sulloway et al. Automated molecular microscopy: the new Leginon system. Journal of Structural Biology 151 (2005), p. 41.

[6] Cheng A et al. High resolution single particle cryo-electron microscopy using beam-image shift. Journal of Structural Biology 204 (2018), p 270.

[7] Some of this work was performed at the National Center for CryoEM Access and Training (NCCAT) and the Simons Electron Microscopy Center located at the New York Structural Biology Center, supported by the NIH Common Fund Transformative High Resolution Cryo-Electron Microscopy program (U24 GM129539,) and by grants from the Simons Foundation (SF349247) and NY State Assembly. 